\title{
Monitoring dynamic electrochemical processes with in situ ptychography
}

\author{
George Kourousias ${ }^{1} \cdot$ Benedetto Bozzini ${ }^{2} \cdot$ Michael W. M. Jones $^{3,4,7} \cdot$ Grant A. Van Riessen $^{5} \cdot$ Simone Dal Zilio $^{6}$. \\ Fulvio Billè ${ }^{1} \cdot$ Maya Kiskinova $^{1} \cdot$ Alessandra Gianoncelli $^{1}$
}

Received: 10 November 2017 / Accepted: 27 February 2018 / Published online: 12 March 2018

(c) The Author(s) 2018. This article is an open access publication

\begin{abstract}
The present work reports novel soft X-ray Fresnel CDI ptychography results, demonstrating the potential of this method for dynamic in situ studies. Specifically, in situ ptychography experiments explored the electrochemical fabrication of Codoped Mn-oxide/polypyrrole nanocomposites for sustainable and cost-effective fuel-cell air-electrodes. Oxygen-reduction catalysts based on Mn-oxides exhibit relatively high activity, but poor durability: doping with Co has been shown to improve both reduction rate and stability. In this study, we examine the chemical state distribution of the catalytically crucial Co dopant to elucidate details of the Co dopant incorporation into the Mn/polymer matrix. The measurements were performed using a custom-made three-electrode thin-layer microcell, developed at the TwinMic beamline of Elettra Synchrotron during a series of experiments that were continued at the SXRI beamline of the Australian Synchrotron. Our time-resolved ptychography-based investigation was carried out in situ after two representative growth steps, controlled by electrochemical bias. In addition to the observation of morphological changes, we retrieved the spectroscopic information, provided by multiple ptychographic energy scans across $\mathrm{Co}_{3}$-edge, shedding light on the doping mechanism and demonstrating a general approach for the molecular-level investigation complex multimaterial electrodeposition processes.
\end{abstract}

Keywords Coherent diffractive imaging · Fresnel CDI · Ptychography $\cdot$ In situ $\cdot$ Oxygen reduction reaction · Electrocatalysis $\cdot$ Fuel cells

George Kourousias

george.kourousias@elettra.eu

Elettra-Sincrotrone Trieste, Trieste, Basovizza, Italy

2 Department of Innovation Engineering, University of Salento, via Monteroni, Lecce, Italy

3 Australian Synchrotron, Melbourne, Australia

4 ARC Centre of Excellence for Advanced Molecular Imaging, La Trobe Institute for Molecular Science, La Trobe University, Melbourne, Australia

5 La Trobe Institute for Molecular Science, La Trobe University, Melbourne, Australia

6 IOM-CNR, Trieste-Basovizza, Trieste, Italy

7 Present Address: Central Analytical Research Facility, Institute for Future Environments, Queensland University of Technology, Brisbane, Australia

\section{Introduction}

Research and development efforts developing battery-based high-energy storage technologies-chiefly grid-support systems and automotive, as well as aerospace electrical propulsion-are impeded by poorly understood, and consequently inefficiently controllable electrochemical phase-formation processes involved both in the fabrication and operation of the devices (Larcher and Tarascon 2014; Kittner et al. 2017). In particular, lack of control over morphology and chemical-state distribution during electrodeposition is the key factor giving rise to damaging of electrodes, electrolytes and separators, ultimately resulting in capacity fade and mechanical failures (Finegan et al. 2015; Yang et al. 2017; Jin et al. 2017). Novel materials and better battery management protocols are being systematically introduced, leading to some progress in the efficiency and reliability of electrochemical energy storage devices. Nevertheless, the pace to success is slower than it could be owing to the prevalence of empirical approaches in technological innovation 
action (Zhang et al. 2017; Liang et al. 2017; Cheng et al. 2017). Further progress in this field requires direct access to relevant physico-chemical information about the systems at relevant space and time scales. Experimental access to localization descriptors of electrochemical phase formation processes is currently possible, thanks to a suite of advanced, mainly synchrotron-based in situ microspectroscopic methods with imaging, topological, chemical and structural capability. Thus, the use of these methods, exhibiting space resolution suitable to address the functionally relevant scales, is starting to gain recognition in the battery community (Wu et al. 2017; Lee et al. 2017). In particular, imaging and structural methods based on X-rays, such as absorption near-edge spectroscopy, fluorescence, diffraction and scattering, are regarded as the cutting-edge tools for the study of buried electrochemical interfaces (Ebner et al. 2013; Lin et al. 2017; Sun et al. 2017). Pioneering investigations in the field, thriving on the complementarity of the approaches, have disclosed a wealth of morphological and chemical modifications of active materials resulting from growth and dissolution processes and have provided unprecedented insight at the submicron scale into complex dynamic interfacial phenomena (e.g., Nagy 2011; Manke et al. 2011; Bozzini et al. 2012a, 2015a). Moreover, advances in lithographic fabrication of cells has allowed the technical limitations of in situ experiments to be overcome, enabling observations of electrode/electrolyte interfaces at normal incidence to the photon beam (Bozzini et al. 2014). Notwithstanding the notable progresses accumulated over the last decade, nanometre space resolution and acquisition rates able to follow chemical kinetics still remain a challenge. The recently proposed microspectroscopic approaches based on coherent diffractive imaging (CDI) have shown that nanometric resolution can be achieved quasi-in situ (Bozzini et al. 2017a) as well as in situ (Kourousias et al. 2016; Bozzini et al. 2017b) and have shown dynamic capabilities that —with appropriate technological development—can be extended down to the millisecond range, opening up the possibility of following chemical kinetics on a scale suitable for dynamic mechanistic studies. The uniqueness of this type of imaging is its capability of overcoming the resolution limits imposed by focusing optics, enabled by the replacement the image-forming lens with an iterative algorithm simultaneously recovering phase and absorption images (Mancuso et al. 2010; Chapman and Nugent 2010). In principle, in CDI the size of the object has to be smaller than the illumination spot, but extended objects can be studied by Fresnel CDI methods-such as Fresnel CDI ptychography (Phillips et al. 2014; Kourousias et al. 2016)-, in which the material under investigation is placed downstream of the focus of a Fresnel zone plate (FZP). Fresnel CDI can also be implemented in the keyhole mode, in which selected portions of extended objects can be studied in detail (Kourousias et al.
2016; Abbey et al. 2008; Zhang et al. 2013). Fresnel-CDI methods based on FZPs are specially suitable for in situ work-in which the precise localization of the active interface is vital-since a real-time holographic image gives the possibility of pre-selecting the region of interest in the direct space, thus combining the advantages of direct and Fourier imaging modes (Bozzini et al. 2017a). Moreover, ptychography can provide space-resolved chemical-state information through the phase and amplitude maps recovered by scanning the photon energy across elemental resonances (Hoppe et al. 2013; Maiden et al. 2013; Kourousias et al. 2016; Bozzini et al. 2017b; Pfeiffer 2018).

In this paper, we report an extension of our pioneering work on dynamic in situ electrochemical ptychography (Kourousias et al. 2016; Bozzini et al. 2017b), proposed as a tool for monitoring morphological and chemical-state changes during phase-formation processes at nanometric length scales. Our previous studies concentrated on the distribution and speciation of Mn during the co-electrodeposition of $\mathrm{Mn}-\mathrm{Co} /$ polypyrrole (PPy) nanocomposites, a promising electrocatalyst for air cathodes in alkaline fuel cells, while in the present research the focus is on the distribution of $\mathrm{Co}$, a dopant of active Mn-oxide ensuring durability under operating conditions. In particular, we have followed representative growth steps from the liquid precursor solution, identifying the onset of unstable growth. The present research confirms the great potential of in situ electrochemical studies with high space-resolution to provide unique insight into interfacial processes involving morphochemical transformations that exhibit functional impact. Ultimately, the information gained through these advanced analytical approaches will allow accurate evaluation of whether specific morphochemical changes resulting from electrodeposition improve or degrade catalytic performance.

\section{Materials and methods}

\section{Ptychography measurements}

Electrochemical growth was followed in situ with Fresnel CDI ptychography at the SXRI beamline of the Australian Synchrotron (van Riessen et al. 2013). The monochromatized beam was shaped into a microprobe with FZP optics (160 $\mu \mathrm{m}$ outer diameter, $30 \mu \mathrm{m}$ diameter of central stop) that was delivered to the cell, placed ca. $0.5 \mathrm{~mm}$ after the focus, yielding a circular illumination of the sample ca. $35 \mu \mathrm{m}$ in diameter. Scattered X-rays were collected with a $2048 \times 2048$ pixel CCD camera placed ca. $32 \mathrm{~cm}$ after the focal spot (Jones et al. 2014). The ptychographic protocol consisted in raster scanning $3 \times 5$ positions separated by steps of $6 \mu \mathrm{m}$, yielding an areal overlap of $82 \%$ (Kourousias et al. 2016). The electrochemical thin-layer transmission 
cell, described in detail in (Bozzini et al. 2017b), was directly mounted on the SXRI sample holder and connected to a VersaSTAT Potentiostat via electrical feedthroughs. The reconstructions were carried out with the NADIA (Davidson et al. 2011) software library, combined with a set of image pre- and post-processing routines developed in Python.

\section{Electrodeposition protocol}

The precursor solution used for the growth of $\mathrm{Mn}-\mathrm{Co} /$ polypyrrole (PPy) nanocomposites has the following composition: $\mathrm{MnCl}_{2} 25 \mathrm{mM}$; $\mathrm{CoCl}_{2} 25 \mathrm{mM}$; pyrrole $0.1 \mathrm{M}$; tetra-butyl-ammonium-perchlorate $0.1 \mathrm{M} ; \mathrm{H}_{2} \mathrm{O} 1 \mathrm{vol} \%$. The electrodeposition protocol consists in a sequence of potentiostatic cycles, explained in (Bozzini et al. 2015b). Briefly, each cycle consists of the following steps (potentials refer to an Au quasi-reference electrode): a pre-step of $1 \mathrm{~s}$ at $-0.3 \mathrm{~V}$ ensures the double-layer relaxation; two oxidising steps of 0.5 and $0.2 \mathrm{~s}$ at $0.9 \mathrm{~V}$ form PPy and Mn(III,IV) ions and a reducing step of $0.5 \mathrm{~s}$ at $-2.1 \mathrm{~V}$ yields $\mathrm{Co}(0)$ and $\mathrm{Mn}(\mathrm{II}) /$ Co(II) oxy-hydroxides.

\section{Results and discussion}

Imaging of selected areas of the working electrode was carried out in situ after two electrodeposition steps, representative of the fabrication of a practical material. The chosen areas are suitable to yield information on the Co chemicalstate distribution resulting from the current-density distribution prevailing in the cell; the current-density distribution is positively correlated with growth rate (Popov et al. 2002). Regarding electrodeposition steps, the first one forms an electrocatalyst film of a thickness in the range relevant to real-life applications, while the second one deposits an incremental amount of materials, that would not affect the practical performance of the catalyst, but allows to singleout morphochemical changes that can yield important mechanistic information. In a recent work on this material, we have investigated the chemical-state distribution of $\mathrm{Mn}-$ the main component of the catalyst - while in this study we shall attack the dynamic morphochemistry of the Co dopant. In view of this, we performed spectroptychographic investigation by recording stacks of ptychographic images at different energies across the $\mathrm{Co}_{3}$-edge $(780 \mathrm{eV})$. Since, as pinpointed in (Kourousias et al. 2016), in the relevant soft X-ray energy range the key components of the growing material (i.e., $\mathrm{Mn}, \mathrm{Co}$ and $\mathrm{C}$ ) have comparable absorption, darker features in the absorption images correspond to the $\mathrm{Mn}-\mathrm{Co} /$ PPy composite aggregates (Henke and Gullikson 1993).

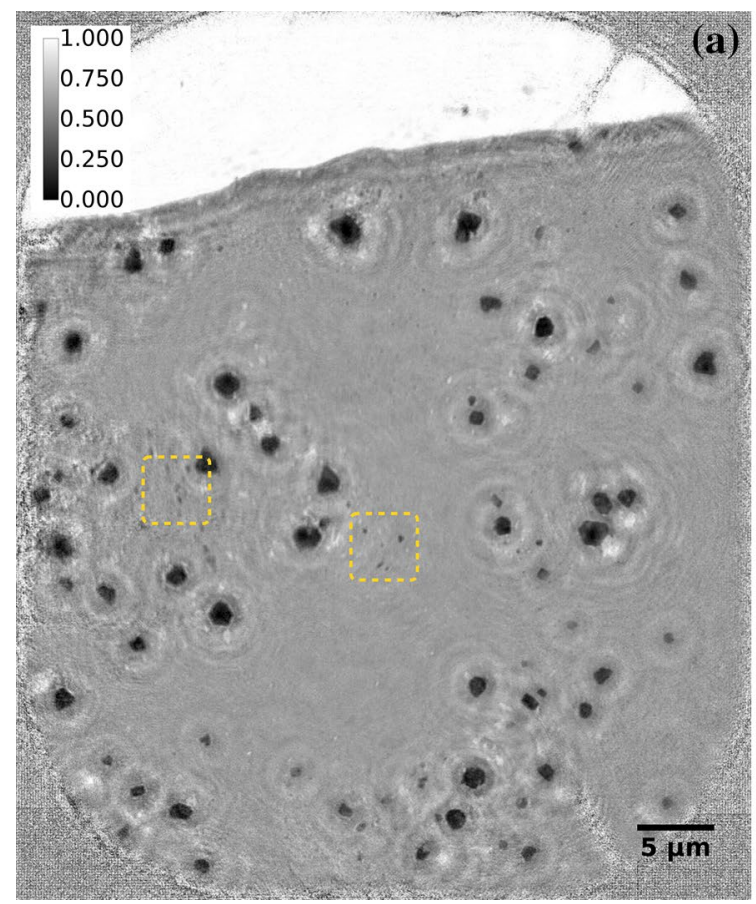

Fig. 1 Image reconstructions from ptychographic scans of a $\mathrm{Mn}-\mathrm{Co} /$ PPy film, measured in situ after 100 growth cycles. Magnitude (a) and Phase (b) images, (b) acquired at $778 \mathrm{eV}$ (Co edge). The raw data

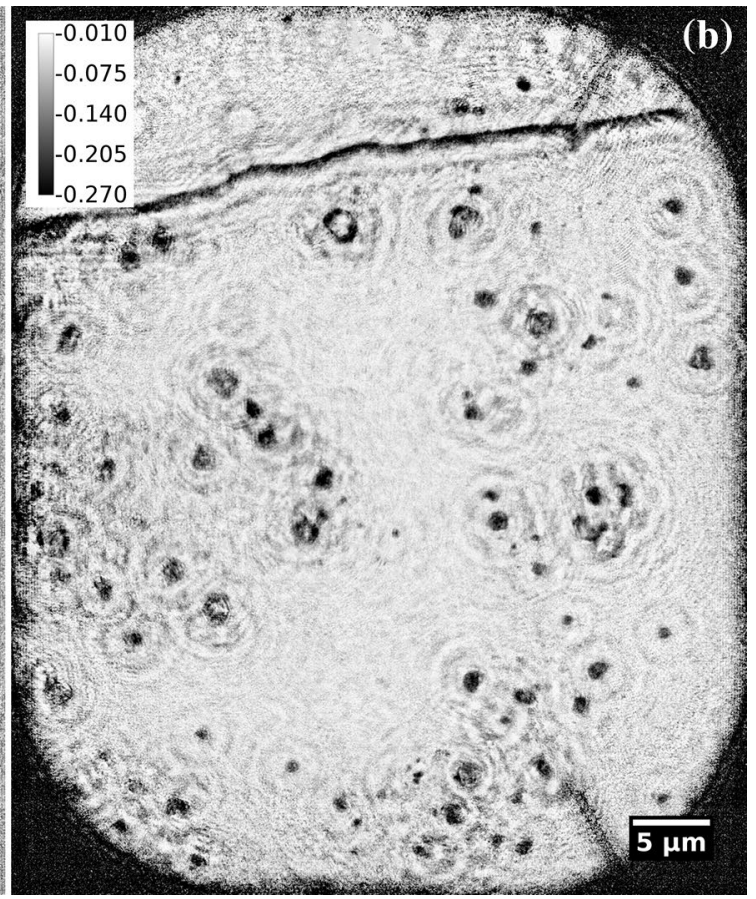

were acquired with $3 \times 5$ scans with $6 \mu \mathrm{m}$ step $\left(47 \times 57 \mu \mathrm{m}^{2}\right)$, shifting the beam energy across the $\mathrm{Co}_{3}$-edge in 16 steps

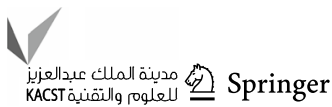




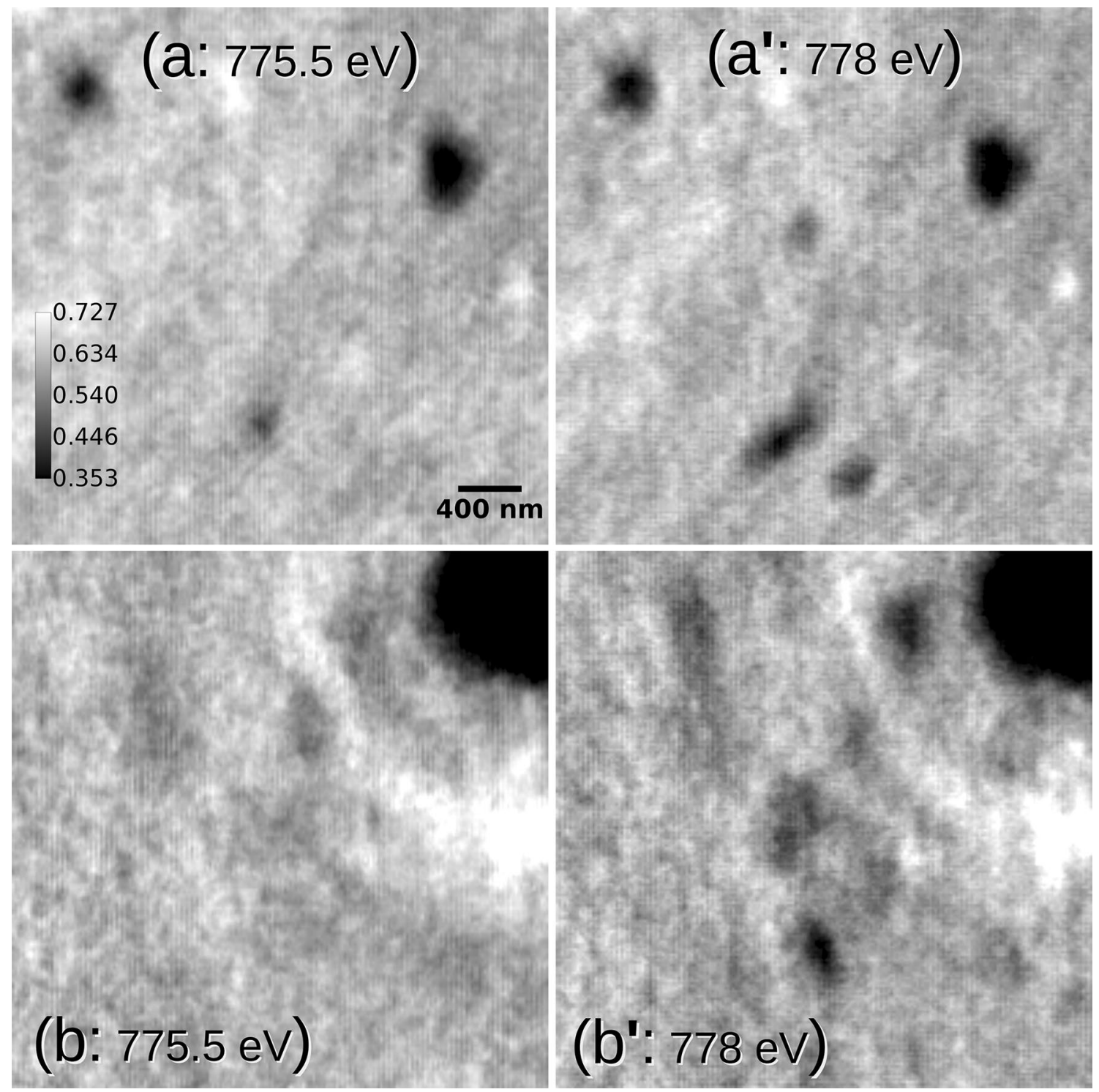

Fig. 2 Reconstructed magnitude images for two $3.3 \times 3.3 \mu \mathrm{m}^{2}$ ROIs, corresponding to the regions highlighted by yellow boxes in Fig. 1a. Images reconstructed employing diffraction data collected below $(775.5 \mathrm{eV}, \mathbf{a}$ and $\mathbf{b})$ and at $\left(778 \mathrm{eV}, \mathbf{a}^{\prime}\right.$ and $\left.\mathbf{b}^{\prime}\right)$ the $\mathrm{Co} \mathrm{L}_{3}$-edge

\section{In situ imaging after thin film growth}

A series of ptychographic scans was acquired on an extended area of the working electrode (ca. $50 \times 50 \mu \mathrm{m}^{2}$ ), after having grown Mn-Co/PPy for 100 cycles: Fig. 1 shows the reconstruction of magnitude (a) and phase (b) images. The data from ptychographic scans recorded at 16 energies across the $\mathrm{Co}_{3}$-edge were employed to generate these images. The selected region encompasses the electrode/electrolyte interface (electrolyte at the top of the images) and corresponds to the zone where the largest growth rate gradient develops (Gianoncelli et al. 2013). Within this extended region, two sub-regions (of dimension ca. $3 \times 3 \mu^{2}$ ) were selected (see Fig. 2) in relatively lower-(Fig. $2 a, a^{\prime}$ ) and higher-current density (Fig. 2b, $b^{\prime}$ ) areas at photon energies of $775.5 \mathrm{eV}$ panels (a) and (b) and at the $\mathrm{Co} \mathrm{L}_{3}$-edge $778 \mathrm{eV}$, panels (a') and $\left(b^{\prime}\right)$, corresponding to the zones highlighted with yellow boxes in Fig. 1a. As the pairs of images in Fig. 2 were acquired below and at the $\mathrm{Co}_{3}$-edge, they also contain elemental contrast. The absorption images acquired at the Co $\mathrm{L}_{3}$-edge [panels $\left(\mathrm{a}^{\prime}\right)$ and $\left(\mathrm{b}^{\prime}\right)$ ] exhibit some dark spots that do not show up in the same area scanned at an energy below the $\mathrm{Co}_{3}$-edge, unequivocally pinpointing that $\mathrm{Co}$ islands have formed during the growth period preceding imaging. Furthermore, one can clearly notice that the density of Cocontaining features is higher in the higher-current density region, in accord with electrochemical nucleation theory (Bozzini et al. 2012b). Comparison of images taken below 

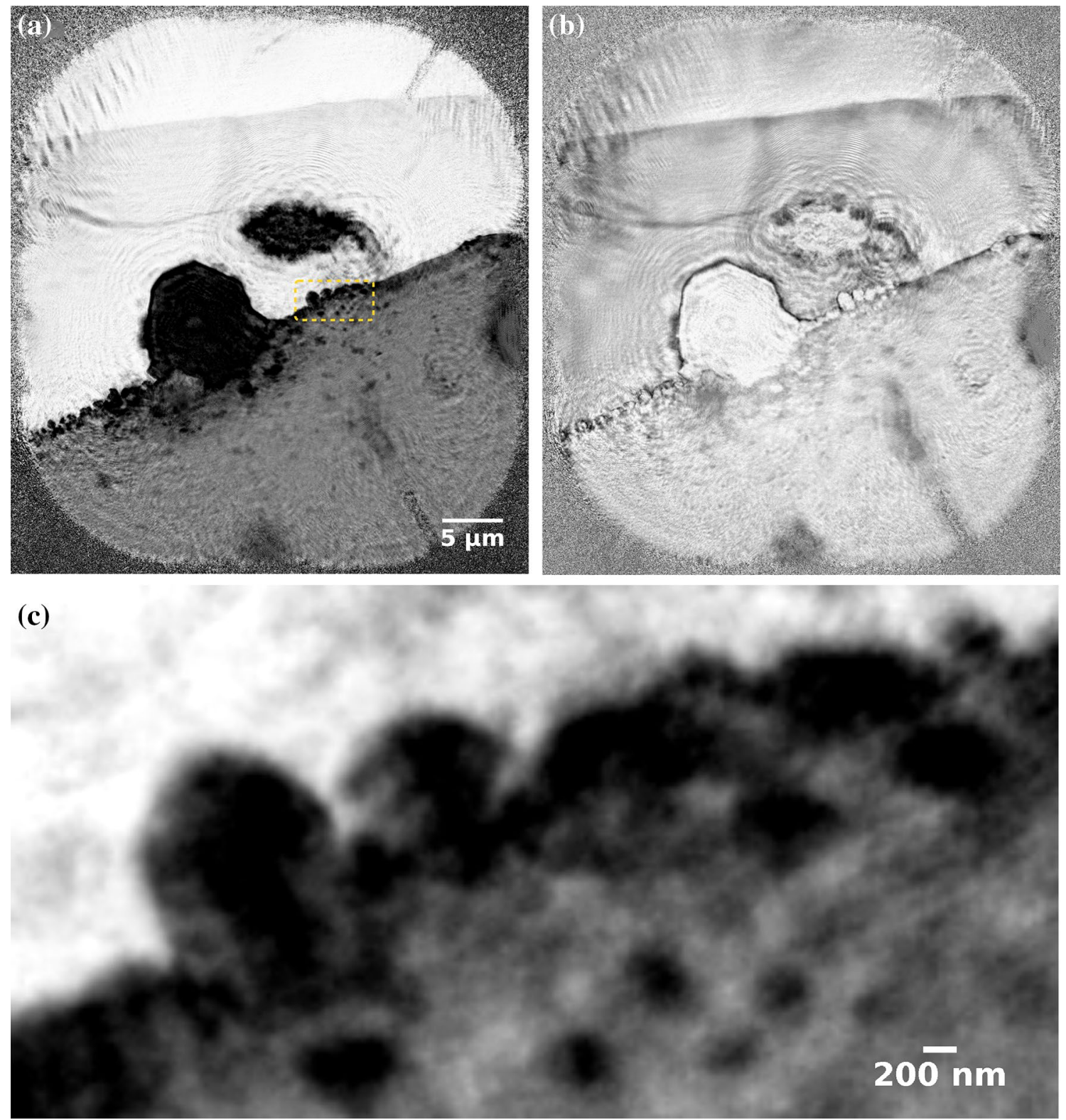

Fig. 3 Reconstructed magnitude (a) and phase (b) images $\left(45 \times 45 \mu^{2}\right)$ of outgrowth features exhibiting characteristic meso- and nano-scale structure. c Detail (see yellow box in a, ROI

and at the absorption edge provides a detailed insight into the Co space distribution within the catalyst matrix, showing that clusters ca. $200 \mathrm{~nm}$ in diameter tend to form. The microscopic capabilities of in situ ptychography are even better appreciated by imaging an electrodeposited feature exhibiting multiple characteristic length scales, such as the dendrites depicted in Fig. 3. Dendrites, like the ones depicted here, are typical unstable growth features that are quite common in electrodeposition and generally correlated with poorer-quality materials. The complementarity of simultaneous magnitude and phase imaging is statistically represented
$6 \times 3 \mu \mathrm{m}^{2}$ ) highlighting nuclei developing at the electrode-electrolyte interface. The reconstructed images employ diffractograms recorded at $778 \mathrm{eV}$

by inspecting Fig. 3a, b: phase imaging allows to visualize the array of outgrowing nuclei as well as the thicker deposit layer developing at the electrode/electrolyte interface in all positions (both the straight horizontal line corresponding to the lithographed $\mathrm{Au}$ current feeder and the bulging dendritic features), while in magnitude images the high absorption of the dendrites reduces the contrast of thinner-projecting objects. Moreover, a classical example of the impact of the exceptional multiscale capability of ptychography for the in situ study of electrochemical phase formation is provided by the comparison of Fig. 3a, c; these micrographs were 

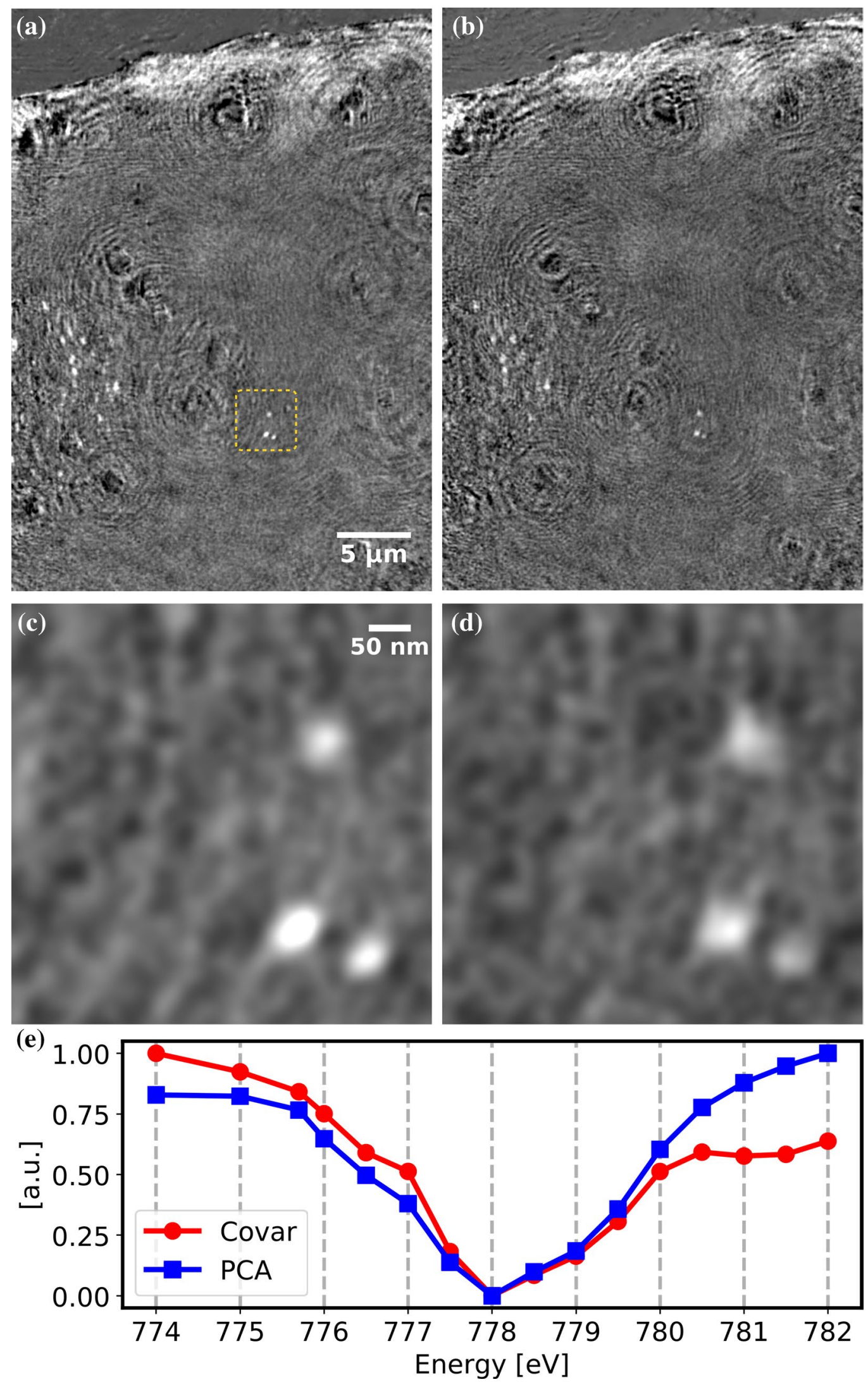

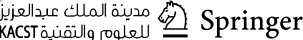


4Fig. 4 Reconstructed magnitude images of the same region depicted in Figs. 1 and 2, obtained by multivariate statistical methods; the brighter colors indicate higher Co presence. The contribution at the Co edge $(778 \mathrm{eV})$ — extracted by covariance (a, c) and PCA (b, d) approaches-emphasizes the Co localization at different scales: a, b ROI $28 \times 39 \mu \mathrm{m}^{2}, \mathbf{c}, \mathbf{d}$ ROI $3.3 \times 3.3 \mu \mathrm{m}^{2} . \mathbf{a}, \mathbf{c}$ are produced through the covariance of a reference spectrum (red in $\mathbf{e}$-acquired on the interface), (b) and its ROI (d) are obtained through PCA (component shown in blue in e). The covariance and PCA methods produce very similar results

derived from the same reconstruction-and consequently from a dataset acquired at a given time under exactly the same operating conditions-and allow to image simultaneously: (1) the whole shape of micrometric dendrites, (2) the formation of submicrometric nuclei and (3) the nanometric structures both in their bulk and at the electrode/electrolyte interface.

To better highlight the areas containing Co aggregates, we employed multivariate analysis approaches-in particular PCA and Covariance analysis, based on the referencing of measured spectra to the spectrum of a pure Co standard-as contrast-enhancing tools. Figure $4 a$, b report reconstructions based on multivariate analysis of a large portion of the extended working-electrode region shown in Fig. 1, while Fig. 4c, d show details of the zone imaged in Fig. 2a, $a^{\prime}$. The results clearly show that the contrast can be notably enhanced with this statistical approach and that even features with characteristic dimensions of a few tens of nanometers can be clearly imaged, allowing us to extend directly the chemical imaging capability down to the dimensional range of the smallest stable nuclei forming in electrocrystallization processes.

\section{In situ imaging after incremental growth}

Further to the assessment of space variations in the distribution of Co after 100 plating cycles, we have endeavoured to follow the amount of the doping element added to the pre-existing deposit structure as a result of applying 10 more growth cycles. Adding nominally $10 \%$ of material is not expected to change the catalytic performance (Bocchetta et al. 2016), but can provide precious mechanistic information in the framework of the DIB model of electrodeposition based on the feedback of electrodeposit morphology on chemical localization (Bozzini et al. 2012c; Lacitignola et al. 2014; Gianoncelli et al. 2015). Thus, after the second growth step, we recorded diffractograms with the same procedure described above, again scanning the energy across the $\mathrm{Co}_{3}$-edge. Figure 5 compares the reconstructed magnitude images at two energies, below $(775.5 \mathrm{eV})$ and at the $\mathrm{Co}_{3}$-edge $(778 \mathrm{eV})$, after 100 and 110 growth cycles. Thus, by comparing the images measured at the $\mathrm{L}_{3}$-edge with those recorded off-edge, the formation of Co-containing features can be spotted. Moreover, differential absorption appearing after the application of the 10 new electrodeposition cycles pinpoints morphology changes in Co distribution. In particular, it can be noticed that on the one hand, pre-electrodeposited crystallites change their shape and on the other hand new nuclei appear. Thanks to chemical sensitivity, ptychographic data encode information about both shape and amount of electrodeposited material, allowing to quantify variations originated by to a given sequence of growth steps. In keeping with our previous observations on Mn (Kourousias et al. 2016; Bozzini et al. 2017b) and with the predictions of the DIB model (in particular Lacitignola et al. 2014), the evolution of the individual Co aggregates shows that global growth is indeed accompanied by local dissolution events.

\section{Spectroscopic ptychography}

The chemical-state distribution can be followed accurately by extracting micro-XAS spectra from selected areas. In this investigation, we have computed micro-XAS from circular regions, ca $500 \mathrm{~nm}$ in diameter, of the images measured after 100 and 110 growth steps (see in Fig. 5). The spectra shown in Fig. 6 exhibit an evident space-dependence of the oxidation state of Co. Moreover, additional growth has a clear effect in some locations. The detailed physico-chemical framework for correlating the chemical state of Co with current-density in metal/PPy composite plating is given in (Bozzini et al. 2014) and extended to alloys in (Bozzini et al. 2015b). To interpret the set of results plotted in Fig. 6, we have to take into account two factors: (a) zones (1) and (2) are representative of intermediate current-density conditions while zones (3) and (4) corresponds to low- and high-current density regions, respectively; (b) owing to the local morphological evolution caused by electrodeposition (Popov et al. 2002), further growth tends to increase the current-density level in high-current density regions (the curvature of deposits tips increases) and to lower it in low-current density ones (increasing screening effect). In (Bozzini et al. 2014) we have shown that the $\mathrm{Co}(\mathrm{II}) / \mathrm{Co}(0)$ ratio correlates with current-density, as a result of the sequence of anodic and cathodic steps adopted for the growth of the relevant class of composites and this scenario is precisely confirmed by the present results. In fact, the most screened region (3) exhibits a higher fraction of elemental Co (main peak close to $778 \mathrm{eV}$ and tendency to further localize the minimum at 778 after 10 additional growth steps). At the opposite limit, the high-current density zone (4) exhibits a clear oxidic spectral component at $779.5 \mathrm{eV}$, that is found to dominate after 110 growth steps. In the intermediate-current density zones (1) and (2) spectrally intermediate conditions are found, with a limited sensitivity on further growth.

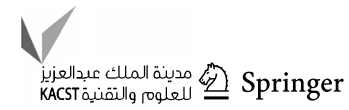



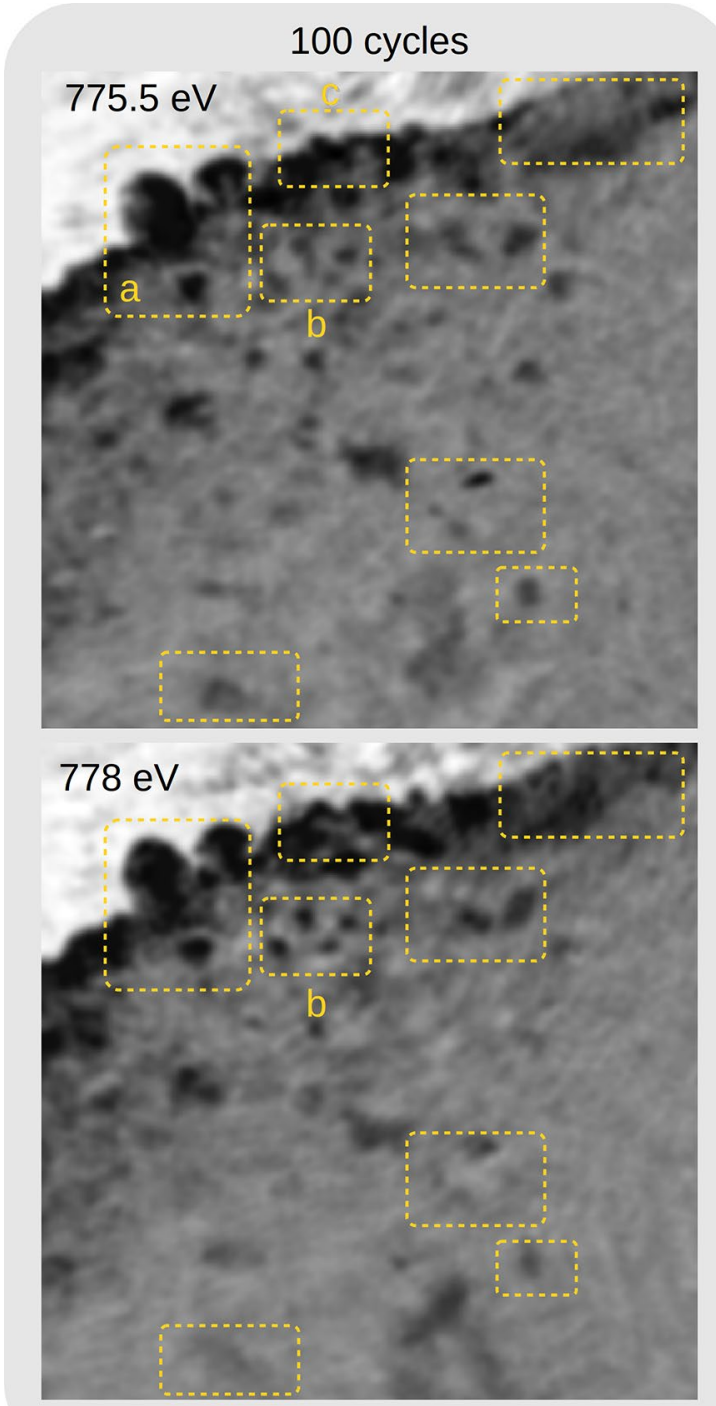

Fig. 5 Reconstructed images (modulus of the principal PCA component, ROI $11 \times 11 \mu \mathrm{m}^{2}$ ) at the electrode/electrolyte interface, measured after 100 and 110 growth cycles. The yellow rectangles highlight regions that exhibit differences resulting either from energy

\section{Conclusions}

This paper reports dynamic spectroscopic ptychography at the $\mathrm{Co}_{3}$-edge performed in situ during the electrodeposition of a Co-doped Mn/PPy nanocomposite ORR electrocatalyst. This is an extension of our previous study on the compositionally prevailing $\mathrm{Mn}$ to dopants, confirming that this approach can provide unique in situ insight at the nanoscale into complex electrochemical phase-formation processes even addressing elements present in low concentrations. The extended nano-imaging capability, obtained by employing multivariate statistical approaches to enhance chemical contract, allows to employ in situ Fresnel CDI for

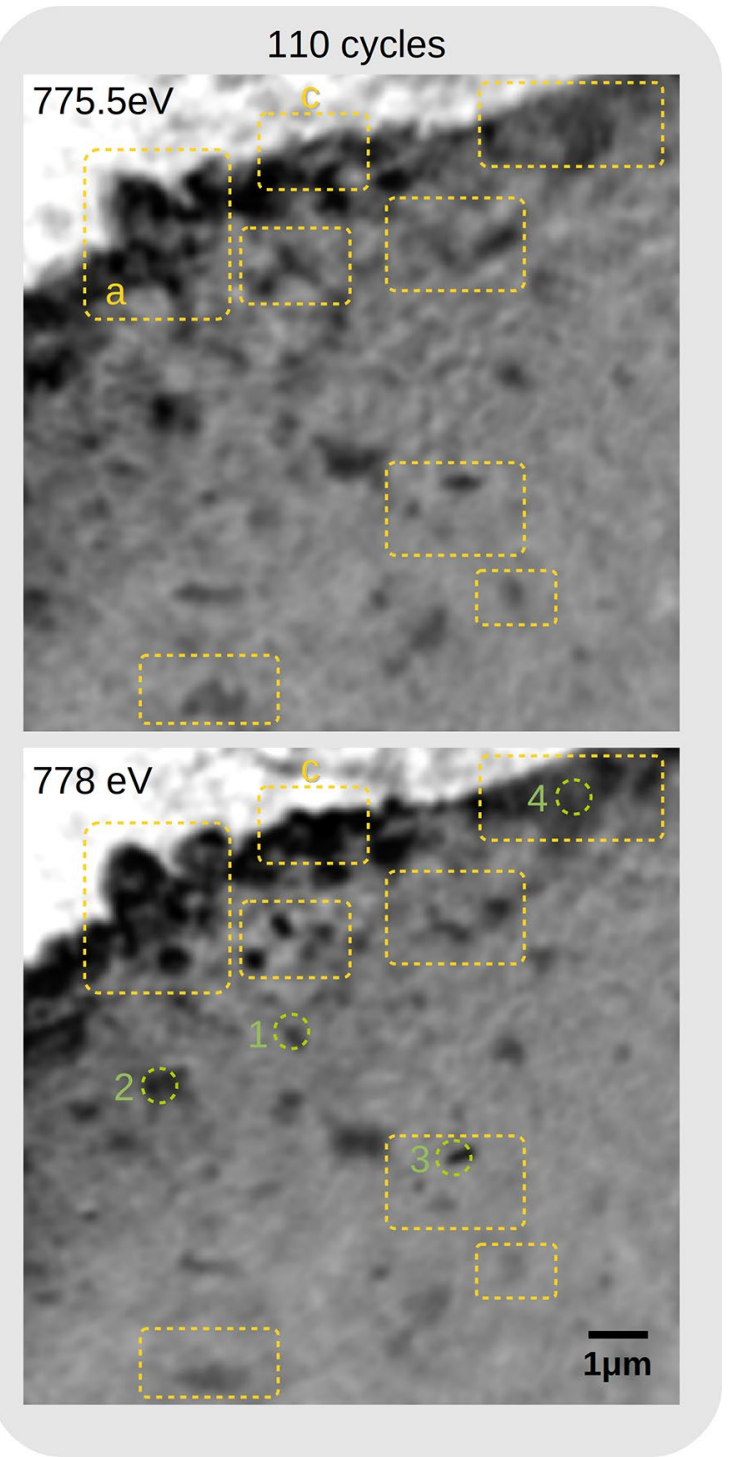

contrast (Co-rich features) or from further growth (electrodeposition of new Co-containing crystallites). Circles associated to a number identify the regions selected for the spectral analyses reported in Fig. 6

the chemical imaging of incipient electrocrystallization processes. The wealth of morphochemical information gleaned by-in situ ptychography calls for a modelling environment able to profit from the raw data collected with high precision in view of developing physico-chemical insight. In this context, the DIB model for electrochemical phase-formation (Bozzini et al. 2012c; Lacitignola et al. 2014; Gianoncelli et al. 2015) in conjunction with parameter-identification tools able to extract compact kinetic data in an automated way (D'Autilia et al. 2017; Sgura et al. 2018) is a promising platform for the development of knowledge-based electrochemical material design and battery control. Even though the specific focus of this paper is on electrodeposition, the 

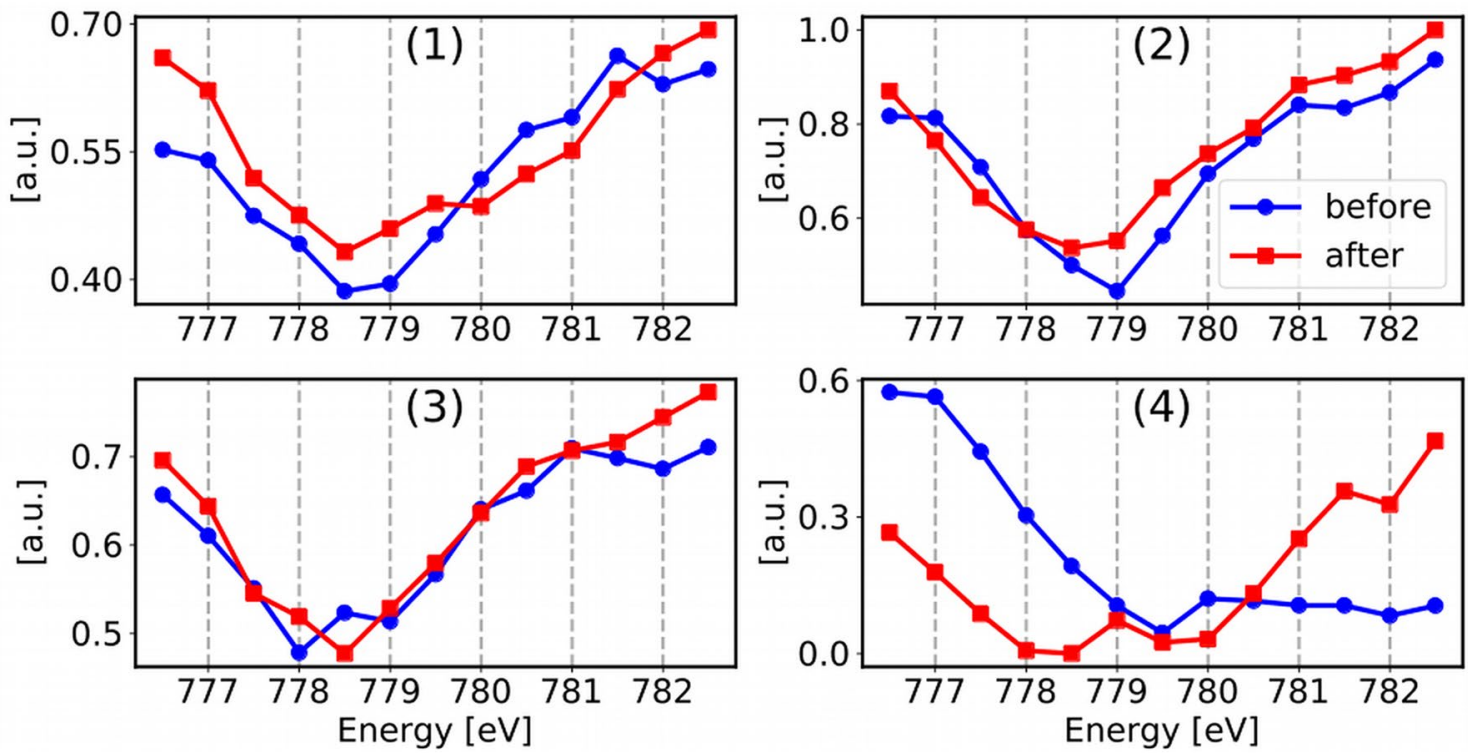

Fig. 6 Micro-XAS spectra extracted from the regions highlighted with yellow circles in Fig. 5. Spectra plotted in red and blue correspond to material formed after 100 and 110 growth cycles, respectively

proposed approach can be naturally extended to any electrochemical material science problem, including — among many others-those relevant to all types of fuel-cells, batteries, solar water-splitting and $\mathrm{CO}_{2}$ reduction processes. However, we note that there are specific sample and photon energy requirements to ensure sufficient $\mathrm{X}$-ray transmission and the relevant spectroscopy can be obtained which may limit the application to a subset of material science problems.

Acknowledgements The authors gratefully acknowledge Dr. Patrizia Bocchetta (Università del Salento, Italy) for the preparation of the electrolyte. This research was undertaken on the soft X-ray imaging (SXRI) beamline at the Australian Synchrotron, Victoria, Australia. Part of the research was supported by the Advanced Integrated Imaging Initiative (AI3) project of Elettra-Sincrotrone Trieste. The microfabrication work was supported by the European Research Infrastructure NFFA-Europe, funded by EU's H2020 framework program for research and innovation.

\section{Compliance with ethical standards}

Conflict of interest There are no conflicts to declare.

Open Access This article is distributed under the terms of the Creative Commons Attribution 4.0 International License (http://creativeco mmons.org/licenses/by/4.0/), which permits unrestricted use, distribution, and reproduction in any medium, provided you give appropriate credit to the original author(s) and the source, provide a link to the Creative Commons license, and indicate if changes were made.

\section{References}

Abbey B, Nugent KA, Williams GJ et al (2008) Keyhole coherent diffractive imaging. Nat Phys 4:394-398. https://doi.org/10.1038/ nphys 896

Bocchetta P, Alemán B, Amati M et al (2016) ORR stability of Mn$\mathrm{Co}$ /polypyrrole nanocomposite electrocatalysts studied by quasi in situ identical-location photoelectron microspectroscopy. Electrochem Commun 69:50-54. https://doi.org/10.1016/j.eleco m.2016.05.018

Bozzini B, Abyaneh MK, Amati M et al (2012a) Soft X-ray imaging and spectromicroscopy: new insights in chemical state and morphology of the key components in operating fuel-cells. Chem Weinh Bergstr Ger 18:10196-10210. https://doi.org/10.1002/ chem. 201201313

Bozzini B, Lacitignola D, Mele C, Sgura I (2012b) Morphogenesis in metal electrodeposition. Note Mat 32(1):7-46

Bozzini B, Lacitignola D, Sgura I (2012c) Spatio-temporal organization in alloy electrodeposition: a morphochemical mathematical model and its experimental validation. J Solid State Electrochem 17:467-479. https://doi.org/10.1007/s10008-012-1945-7

Bozzini B, Gianoncelli A, Bocchetta P et al (2014) Fabrication of a sealed electrochemical microcell for in situ soft X-ray microspectroscopy and testing with in situ co-polypyrrole composite electrodeposition for Pt-free oxygen electrocatalysis. Anal Chem 86:664-670. https://doi.org/10.1021/ac403004v

Bozzini B, Altissimo M, Amati M, Bocchetta P, Gianoncelli A, Gregoratti L, Kourousias G, Mancini L, Mele C, Kiskinova M (2015a) In situ and ex situ X-ray microspectroelectrochemical methods for the study of zinc-air batteries. In: Reedijk J (ed) Elsevier Reference Module in Chemistry, Molecular Sciences and Chemical Engineering. Elsevier, Waltham, MA. https://doi. org/10.1016/B978-0-12-409547-2.11452-0

Bozzini B, Bocchetta P, Gianoncelli A et al (2015b) Electrodeposition and ageing of Mn-based binary composite oxygen reduction reaction electrocatalysts. ChemElectroChem 2:1541-1550. https://doi. org/10.1002/celc. 201500138 
Bozzini B, Kourousias G, Bedolla DE, Gianoncelli A (2017a) Chemical-state evolution of $\mathrm{Ni}$ in $\mathrm{MnNi}$ /polypyrrole nanocomposites under bifunctional air electrode conditions, investigated by quasi-in situ multi-scale soft X-ray absorption spectroscopy. Mater Today Energy 6:154-163. https://doi.org/10.1016/j.mtene r.2017.09.013

Bozzini B, Kourousias G, Gianoncelli A et al (2017b) Soft X-ray ptychography as a tool for in operando morphochemical studies of electrodeposition processes with nanometric lateral resolution. J Electron Spectrosc Relat Phenom 220:147

Chapman HN, Nugent KA (2010) Coherent lensless X-ray imaging. Nat Photonics 4:833-839. https://doi.org/10.1038/nphoton.2010.240

Cheng X-B, Zhao M-Q, Chen C et al (2017) Nanodiamonds suppress the growth of lithium dendrites. Nat Commun 8:336. https://doi. org/10.1038/s41467-017-00519-2

D’Autilia MC, Sgura I, Bozzini B (2017) Parameter identification in ODE models with oscillatory dynamics: a Fourier regularization approach. Inverse Problems 33(12). https://doi.org/10.1088/13616420/aa9834

Davidson N, Abbey B, Quiney HM, Julius TD, Allman B, Jones MWM, Putkunz CT, Torrance A, Wittler H, Carroll A et al (2011) NADIA Software Project; ARC Centre of Excellence for Coherent X-ray Science. http://www.coecxs.org/joomla/index.php/researchand-projects/nadia-software-project.html. Accessed 10 Feb 2016

Ebner M, Marone F, Stampanoni M, Wood V (2013) Visualization and quantification of electrochemical and mechanical degradation in Li ion batteries. Science 342:716-720. https://doi.org/10.1126/ science. 1241882

Finegan DP, Scheel M, Robinson JB et al (2015) In-operando highspeed tomography of lithium-ion batteries during thermal runaway. Nat Commun 6:7924. https://doi.org/10.1038/ncomms7924

Gianoncelli A, Kaulich B, Kiskinova M et al (2013) Fabrication and testing of an electrochemical microcell for in situ soft X-ray microspectroscopy measurements. J Phys Conf Ser 425:182010. https://doi.org/10.1088/1742-6596/425/18/182010

Gianoncelli A, Sgura I, Bocchetta P et al (2015) High-lateral resolution $\mathrm{X}$-ray fluorescence microspectroscopy and dynamic mathematical modelling as tools for the study of electrodeposited electrocatalysts. X-Ray Spectrom 44:263-275. https://doi.org/10.1002/ xrs. 2617

Henke BL, Gullikson EM (1993) X-ray interactions: photoabsorption, scattering, transmission, and reflection at $E=50-30,000 \mathrm{eV}$, $Z=1-92$. At Data Nucl Data Tables 54:181-342. https://doi. org/10.1006/adnd.1993.1013

Hoppe R, Reinhardt J, Hofmann G et al (2013) High-resolution chemical imaging of gold nanoparticles using hard X-ray ptychography. Appl Phys Lett 102:203104. https://doi.org/10.1063/1.4807020

Jin Y, Zhou G, Shi F et al (2017) Reactivation of dead sulfide species in lithium polysulfide flow battery for grid scale energy storage. Nat Commun 8:462. https://doi.org/10.1038/s41467-017-00537-0

Jones MWM, Dearnley MK, van Riessen GA et al (2014) Rapid, low dose X-ray diffractive imaging of the malaria parasite Plasmodium falciparum. Ultramicroscopy 143:88-92. https://doi. org/10.1016/j.ultramic.2013.09.003

Kittner N, Lill F, Kammen DM (2017) Energy storage deployment and innovation for the clean energy transition. Nat Energy 2:17125. https://doi.org/10.1038/nenergy.2017.125

Kourousias G, Bozzini B, Gianoncelli A et al (2016) Shedding light on electrodeposition dynamics tracked in situ via soft X-ray coherent diffraction imaging. Nano Res 9:2046-2056. https://doi. org/10.1007/s12274-016-1095-9

Lacitignola D, Bozzini B, Sgura I (2014) Spatio-temporal organization in a morphochemical electrodeposition model: analysis and numerical simulation of spiral waves. Acta Appl Math 132:377389. https://doi.org/10.1007/s10440-014-9910-3
Larcher D, Tarascon J-M (2014) Towards greener and more sustainable batteries for electrical energy storage. Nat Chem 7:19-29. https:// doi.org/10.1038/nchem.2085

Lee J, Dupre N, Avdeev M, Kang B (2017) Understanding the cation ordering transition in high-voltage spinel Li Ni $0.5 \mathrm{Mn} 1.5 \mathrm{O} 4$ by doping Li instead of Ni. Sci Rep 7:6728. https://doi.org/10.1038/ s41598-017-07139-2

Liang X, Pang Q, Kochetkov IR et al (2017) A facile surface chemistry route to a stabilized lithium metal anode. Nat Energy 2:17119. https://doi.org/10.1038/nenergy.2017.119

Lin F, Liu Y, Yu X et al (2017) Synchrotron X-ray analytical techniques for studying materials electrochemistry in rechargeable batteries. Chem Rev. https://doi.org/10.1021/acs.chemrev.7b00007

Maiden AM, Morrison GR, Kaulich B et al (2013) Soft X-ray spectromicroscopy using ptychography with randomly phased illumination. Nat Commun 4:1669. https://doi.org/10.1038/ncomms2640

Mancuso AP, Gorniak T, Staier F et al (2010) Coherent imaging of biological samples with femtosecond pulses at the free-electron laser FLASH. New J Phys 12:035003. https://doi.org/10.1088/13672630/12/3/035003

Manke I, Markötter H, Tötzke C et al (2011) Investigation of energyrelevant materials with Synchrotron X-rays and neutrons. Adv Eng Mater 13:712-729. https://doi.org/10.1002/adem.201000284

Nagy Z (2011) Electrochemistry at synchrotrons. J Solid State Electrochem 15:1679-1695. https://doi.org/10.1007/s10008-010-1242-2

Pfeiffer F (2018) X-ray ptychography. Nat Photonics 12:9-17. https:// doi.org/10.1038/s41566-017-0072-5

Phillips NW, Putkunz CT, Van Riessen G et al (2014) Ptychographic Fresnel coherent diffraction tomography at the nanoscale. Int J Mater Res 105:655-663. https://doi.org/10.3139/146.111065

Popov K, Grgur B, Djokić SS (2002) Fundamental aspects of electrometallurgy. Springer Science \& Business Media, New York

Sgura I, Lawless AS, Bozzini B (2018) Parameter estimation for a morphochemical reaction-diffusion model of electrochemical pattern formation. Inverse Problems in Science \& Engineering

Sun K, Zhao C, Lin C-H et al (2017) Operando multi-modal Synchrotron investigation for structural and chemical evolution of cupric sulfide (CuS) additive in Li-S battery. Sci Rep 7:12976. https:// doi.org/10.1038/s41598-017-12738-0

van Riessen GA, Junker M, Phillips NW, Peele AG (2013) A soft X-ray beamline for quantitative nanotomography using ptychography. In: Lai B (ed). Proceedings vol 8851, X-Ray Nanoimaging: Instruments and Methods, p 885117. https://doi.org/10.1117/12.20272 11

Wu J, Yang S, Cai W et al (2017) Multi-characterization of $\mathrm{LiCoO}_{2}$ cathode films using advanced AFM-based techniques with high resolution. Sci Rep 7:11164. https://doi.org/10.1038/s41598-01711623-0

Yang J, Hu C, Wang H, Yang K, Liu JB, Yan H (2017) Review on the research of failure modes and mechanism for lead-acid batteries. Int J Energy Res 41:336-352. https://doi.org/10.1002/er.3613

Zhang B, Seaberg MD, Adams DE et al (2013) Full field tabletop EUV coherent diffractive imaging in a transmission geometry. Opt Express 21:21970-21980

Zhang N, Cheng F, Liu J et al (2017) Rechargeable aqueous zinc-manganese dioxide batteries with high energy and power densities. Nat Commun 8:405. https://doi.org/10.1038/s41467-017-00467-x

Publisher's Note Springer Nature remains neutral with regard to jurisdictional claims in published maps and institutional affiliations. 